\title{
Die Zeit ist reif
}

\author{
Gerhard Vigener
}

\begin{abstract}
Trotz schwieriger Rahmenbedingungen müssen die Gesellschaft und die Behindertenhilfe künftig die Kompetenz behinderter Menschen stärker berücksichtigen.
\end{abstract}

Ich erinnere mich gerne an unseren Enthusiasmus, mit dem wir 2003 das »Europäische Jahr der Menschen mit Behinderung « begangen haben. Motto dieses besonderen Jahres war: »Nicht über uns ohne uns!« Zusammen mit der Lebenshilfe Baden-Württemberg habe ich mich damals in meiner früheren Funktion als Verbandsdirektor des Landeswohlfahrtsverbandes Baden bei einer Fotoausstellung engagiert, die Menschen mit Behinderung in Alltagssituationen nichtbehinderter Menschen zeigte: am Steuer eines großen Autos, am Arbeitsplatz, als Pfarrer im Gottesdienst, als glückliches Hochzeitspaar. Diese Bilder sollten die Botschaft vermitteln: »Wir sind so wie ihr, wir haben Kompetenzen, wir erfreuen uns unseres Lebens, wir wollen und können etwas leisten, nehmt uns, so wie wir sind und lasst uns teilnehmen an Eurem Alltag!«

Bei den Eröffnungsveranstaltungen dieser Wanderausstellung haben wir dann immer wieder gerade auch auf die Kompetenzen von Menschen mit Behinderung hingewiesen. Sie produzieren in Werkstätten Möbel, mit denen wir leben, als Zulieferer der Automobilindustrie sind sie am Bau unserer Autos beteiligt, selbst die Medizintechnik lässt in Werkstätten für Menschen mit Behinderung produzieren und montieren. »Grüne Gruppen« pflegen öffentliche Grünanlagen und private Gärten, Werkstätten haben teilweise sogar eigene Weinberge, in denen Menschen mit Behinderung vom Rebschnitt bis zur Lese Beschäftigung haben.

Das Europäische Jahr hat uns diese Kompetenzen vor Augen geführt. Haben wir die Botschaft aufgenommen und uns ernsthaft mit Konsequenzen befasst, die daraus zu ziehen wären? Gewiss: Es gibt viele Symposien und Kongresse, auf denen über Ambulantisierung und Persönliches Budget diskutiert wird. Ein erster großer Erfahrungsbericht über das Persönliche Budget »im Alltag « kommt allerdings noch aus den Niederlanden; dort gibt es heute über 15.000 geistig behinderte Menschen, die sich ihre gesellschaftliche Teilhabe über ein Persönliches Budget selbst organisieren. Bei uns haben wir trotz einer guten rechtlichen Grundlage das Modellstadium des Persönlichen Budgets noch nicht verlassen. Es werden weiter Wohnheime und Werkstätten gebaut, Komplexeinrichtungen saniert, Heilpädagogische Heime in der Trägerschaft der Sozialhilfeträger belassen, die damit ihre Arbeitsplätze mehrfach sichern, als gäbe es die Botschaft von 2003 nicht.

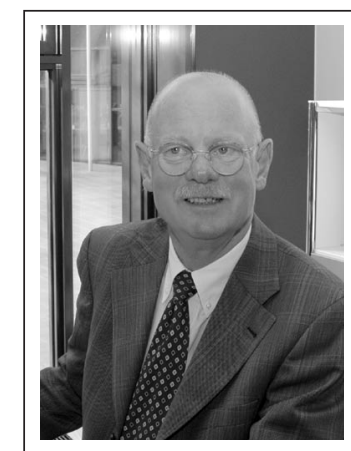

Prof. Dr. jur. Gerhard Vigener ist seit Mai 2005 an der privaten Fachhochschule der SRH-Learnlife GmbH in Heidelberg tätig. Seine Schwerpunkte sind Jugendhilferecht, Sozialhilferecht, Hilfesysteme für Menschen mit Behinderung, Rehabilitationsrecht. Er war von 1981 bis 1985 in Karlsruhe Leiter des Landesjugendamtes Baden, dann bis 2001 Leiter des Landessozialamtes beim Landeswohlfahrtsverband $\mathrm{Ba}$ den und von 2001 bis zur Auflösung im Zuge der Verwaltungsreform in Baden-Württemberg Ende 2004 Verbandsdirektor des Landeswohlfahrtsverbandes Baden. Der hier veröffentlichte Beitrag beruht auf einem Vortrag beim Symposium »Der Kunde stimmt mit den Füßen $a b-V o n$ der Institution zum Dienstleister! «am 16. und 17. Oktober 2006 in Heidelberg.

E-Mail Gerhard.Vigener@fh-heidelberg.de

\section{Schwierige Rahmenbedingungen für einen grundsätzlichen Wechsel}

Die Rahmenbedingungen für einen grundsätzlichen Wechsel in der Einstellung zu Menschen mit Behinderung, in der Begleitung und Förderung von Menschen mit Behinderung sind heute allerdings auch außerordentlich schwierig:

1. Demografisch bedingt steigt die Zahl vor allem der 1. Menschen mit geistiger sowie geistiger und gleichzeitig schwer körperlicher Behinderung auch in den kommenden Jahren weiter. Der Deutsche Verein für öffentliche und private Fürsorge geht auf der Grundlage einer Untersuchung von consens $\mathrm{GmbH}$ davon aus, dass allein von 2002 bis 2007 die Zahl der Menschen, die Eingliederungshilfe im Betreuten Wohnen, in Wohnheimen und Werkstätten erhalten, von 417.000 auf 492.000 steigen wird, also um 18 Prozent. Unter Hinzurechnung behinderter Kinder in einer Tageseinrichtung, in Schul- oder Berufsausbildung oder behinderter Menschen in Kliniken können wir heute von fast 600.000 Menschen ausgehen, die Eingliederungshilfe nach SGB XII bekommen. Bei einem unveränderten Anteil an 
schwerbehindert Geborenen in jedem Geburtsjahrgang die Vergangenheit zeigt, dass wir von dieser Annahme ausgehen müssen - und einer restlichen Lebenserwartung der ältesten vollen Jahrgänge behinderter Menschen (ab 1946) von sicherlich 15 Jahren dürfen im Jahre 2020 über eine Million Menschen mit Behinderung unter uns leben, die nach bisherigem Recht Eingliederungshilfe für Menschen mit Behinderung erhalten könnten.

2. Überörtliche Träger der Sozialhilfe gehen davon aus, dass bei unveränderten Rahmenbedingungen die Nettoausgaben für die Eingliederungshilfe bundesweit von 9,2 Milliarden Euro im Jahre 2002 auf 12, 2 Milliarden Euro im Jahre 2007 steigen werden. Auch dieser Anstieg wird sich allein durch die Zunahme der Fallzahlen in den nachfolgenden Jahren weiter fortsetzen. Dabei sind Personalkostensteigerungen oder von manchen Trägern großer Einrichtungen geforderte Personalverbesserungen nicht berücksichtigt.

3 Moderne Medizin und Einstellung von Eltern Un3. geborener bewirken, dass in den jüngeren Jahrgängen behinderter Menschen der Anteil der ausschließlich geistig behinderten Menschen abnimmt und der Anteil der mehrfach behinderten, geistig und körperlich behinderten Menschen stark zunimmt. Der Unterstützungsbedarf steigt, die zuvor erwähnten Kompetenzen gehen im Einzelfall zurück.

1 Der erste volle Geburtsjahrgang geistig behinderter 4. Menschen erreicht in diesem Jahre 2006 das 60. Lebensjahr. Viele dieser Menschen scheiden derzeit schon aus dem Arbeitsbereich der Werkstatt für Menschen mit Behinderung aus. Sie benötigen eine Tagesstruktur, die bis dahin die Werkstatt geboten hat. Sie werden im übrigen zunehmend pflegebedürftig und brauchen ein adäquates, behindertengerechtes Pflegeangebot, das üblicherweise in der bisherigen Altenpflege nicht gegeben ist.

5 Die Werkstätten für behinderte Menschen sind vor - allem in ihren industriellen Fertigungen einem starken Konkurrenzdruck aus den osteuropäischen Ländern ausgesetzt. Es wird in einigen Bereichen immer schwerer, neue Aufträge zu akquirieren. Damit bleiben nicht nur Beschäftigung, sondern auch Werkstatterlöse aus. Manche Werkstätten sind kaum noch in der Lage, ihren behinderten Mitarbeitern einen Werkstattlohn zu zahlen, der auch Arbeitsanreiz darstellen kann.

6. Eingliederungshilfe für Menschen mit Behinderung - wird aus Landes- und Kommunalhaushalten finanziert. Nachdem die Bundesrepublik drei Jahre lang die Euro-Stabilitätskriterien nicht eingehalten hat und in der Zwischenzeit bei einem Schuldenstand von einer Billion 470 Millliarden Euro angekommen ist (mit einer jährlichen Zinslast von über 60 Milliarden Euro), wird auch der großen Koalition in Berlin, den Landesregierungen und Kommunen trotz der verbesserten Wirtschaftslage künftig nichts anderes übrig bleiben als eine konsequente Sparpolitik.

\section{Trotz knapper Kassen den Umbruch wagen}

Mehr Menschen mit Behinderung, die Eingliederungshilfe erwarten, neue Aufgaben und Herausforderungen für die Behindertenhilfe bei zumindest nicht mehr steigenden finanziellen Ressourcen, die die Bundesländer und Kommunen für die Finanzierung der Eingliederungshilfe aufbringen werden — das sind die Rahmenbedingungen für einen grundsätzlichen Richtungswechsel in der Behindertenhilfe! Da ist kein zusätzliches Geld beispielsweise für Belohnungssysteme bei einem Umstieg vorhanden! Aber wie kann bei knappen Ressourcen ein solcher Richtungswechsel aussehen, der gleichzeitig sicherstellen muss, dass die gesetzlichen Ansprüche auch neuer Leistungsberechtigter erfüllt werden? Eine Kontingentierung der Eingliederungshilfe dürfte weder von den Akteuren gewollt, noch rechtlich möglich sein. Wir wollen keinen »closed shop« für die bisherigen Leistungsberechtigten, der keine Neuzugänge zulässt. Die Leistungen müssen noch genauer dem Bedarf des Einzelnen entsprechen und ihn im Mittelpunkt des Bemühens sehen; der Leistungsempfänger und sein Lebensumfeld müssen vor allem gestärkt werden!

1 Was die Jugendhilfe schon in den 70er Jahren des 1. vergangenen Jahrhunderts mit der »Heimkampagne« auf den Weg gebracht hat, muss endlich auch vor allem für junge Menschen mit geistiger Behinderung Regelleistung werden: fachliche Familienpflege und Wohngruppe im bisherigen Umfeld statt Heimunterbringung! Es war ein großer Fortschritt in der Hilfe für geistig behinderte junge Menschen, dass in den Gemeinden für sie flächendeckend ein besonderes Schulsystem aufgebaut wurde, das auch von ihren Eltern akzeptiert wurde. Vielfach waren es die Elterninitiativen selbst, die den Aufbau und Ausbau des Sonderschulwesens gefordert haben. Es war dann ein weiterer noch größerer Fortschritt, dass heute in Außengruppen und Außenklassen und in Einzelintegration behinderte und nicht behinderte Kinder gemeinsam lernen und aufwachsen. Was passiert, wenn diese behinderten jungen Menschen in die Pubertät kommen und wie viele nicht behinderte Jugendliche erhebliche Schwierigkeiten mit ihren Eltern haben, wenn sie »verhaltensauffällig «, wenn sie »aggressiv« werden und ihre Eltern, die sich schon viele Jahre aufopfernd um sie bemüht haben, keine Kraft mehr haben und resignieren? Nach einigen Versuchen mit familienentlastenden Diensten ist die Heimunterbringung in einer »Komplexeinrichtung « mit Schule die Lösung der Wahl. Es handelt sich sehr oft um junge Menschen, die in ihrer Herkunftsschule und im Freundeskreis nur wenig Probleme haben. In der »Komplexeinrichtung « werden sie dann, aus dem bisherigen Umfeld herausgerissen, erst recht »verhaltensauffällig « — ich habe großes Verständnis für diese jungen Menschen. Die Antwort auf die »Pubertätsprobleme « muss vor Ort gefunden werden, nicht durch eine Verpflanzung über viele Kilometer hinweg! Der Besuch der vertrauten Schule und der Freundeskreis müssen erhalten werden. Daher sollten dringend fachliche Familienpflegestellen und Außengruppen für jugendliche behinderte Menschen, sei es angegliedert an das örtliche Wohnheim, sei es als Pflegestelle in Kooperation mit einem Wohnheim, eingerichtet werden. Ziel einer solchen Hilfe vor Ort kann die Rückkehr in die Familie, kann aber auch eine spätere Verselbstständigung sein.

2. Über 40.000 Menschen mit Behinderung leben heute im Ambulant Betreuten Wohnen. Die meisten von ihnen sind psychisch krank, nicht aber geistig behindert. Von den geistig behinderten Menschen, die 
außerhalb der Herkunftsfamilie leben, sind es höchstens zehn Prozent, die ambulant betreut wohnen. Dieser Anteil ist erheblich steigerbar! Dabei denke ich besonders an die Heimbewohner in Hilfebedarfsgruppe 1 oder 2. Voraussetzung wäre allerdings, auch im Betreuten Wohnen den Hilfebedarfsgruppen entsprechende Maßnahmepauschalen zu haben. Ob in diesem Zusammenhang ein Anreiz für die Anbieter stationärer Hilfeangebote sinnvoll und möglich ist, kann diskutiert werden. Meine Meinung dazu ist, dass sich diese Anbieter auch ohne einen finanziellen Anreiz der Verselbständigung ihrer Klienten öffnen sollten! Im übrigen darf das ambulant betreute Wohnen bisheriger Form nur ein Übergangsstadium sein! In den meisten Fällen ist die »Sachleistung «, wie sie das Ambulant Betreute Wohnen darstellt, nicht gerechtfertigt. Warum keine Geldleistung, warum kein Persönliches Budget?

\section{Ich habe die Idee des Persönlichen Budgets von 3. Anfang an für zielführend gehalten! Wir sind in der derzeitigen Modellphase, die eine komfortable Rechts- grundlage hat, allerdings sehr zurückhaltend und zöger- lich. Trauen wir den Menschen mit Behinderung doch zu wenig zu? Die Budgetverordnung lässt befürchten, dass wir Budgetassistenten mit großen buchhalterischen Kom- petenzen benötigen, um das Budget im Einzelnen zu kal-}

In der industriellen Fertigung wird dies sicher kaum der Fall sein, aber wie steht es mit Dienstleistungen? Einige der vielen behinderten Mitarbeiter von »grünen Gruppen « könnten sicher mit Förderung nach dem Schwerbehindertenrecht im Landschaftsbau arbeiten. Wie steht es mit dem Wirtschaftsbereich von Krankenhäusern, Pflegeheimen, mit der Gastronomie? Das Outsourcing dieser Wirtschaftsbereiche hat vielfach dazu geführt, dass der Tarifvertrag für den öffentlichen Dienst kein Maßstab mehr ist. Minderleistungsausgleich und niedrigere Löhne, letztlich also »Kombilöhne « im Rahmen der Behindertenhilfe machen die Beschäftigung von Menschen mit Behinderung auf diesem Teil des allgemeinen Arbeitsmarktes für alle Seiten interessant. Das kann bis zu haushaltsnahen Dienstleistungen gehen, einem Feld, das bei der Alterung unserer Gesellschaft eine beachtliche $\mathrm{Zu}$ kunftsperspektive hat!

5 Die Finanzierung der klassischen stationären Be- hindertenhilfe muss neu geordnet werden. Elisabeth Wacker zeigt mit ihrem Projekt »Perle« einen interessanten, wenn auch mühsamen Weg auf! Dieser Weg geht deshalb in die richtige Richtung, weil er von der institutionsbezogenen zur klientenorientierten Vergütung führt. Das SGB XII bereitet einen radikaleren Umstieg zur klientenorientierten Vergütung vor, ohne diesen Umstieg in letzter Konsequenz zu regeln. Hilfeempfängergruppen und Leistungstypen versetzen uns heute in die Lage, einen Katalog mit einer Vielzahl von typisierten Bedarfslagen zur Teilhabe von Menschen mit Behinderung aufzustellen. Diese Bedarfslagen können wiederum

kulieren und abzurechnen. In der Pflege gibt es ein Beispiel für ein einfaches, praktikables Budget, das »Pflegegeld «. Wir brauchten in der Hilfe für Menschen mit Behinderung möglicherweise mehr als die drei Stufen des Pflegegeldes. Wir sollten die Beträge des Budgets ableiten von den Maßnahmepauschalen in der Heimbetreuung oder im Ambulant Betreuten Wohnen, ohne dass sie die volle Höhe dieser Pauschalen erreichen. Die Differenz ist der »Preis der Selbständigkeit«! Vor einigen Jahren hat der heute nicht mehr existierende Landeswohlfahrtsverband Baden als überörtlicher Träger der Sozialhilfe ein »Pflegegeld « für die Aufnahme von Menschen mit Behinderung in Geschwisterfamilien eingeführt. Es bestand jedenfalls im Entgelt für die Betreuung aus einer Pauschale. Uns war damals der »Outcome « wichtig, die Qualität der Begleitung, nicht aber der exakte Nachweis einzelner Betreuungsstunden. Das Budget sollte daher stärker pauschaliert werden! Der Nachweis der Selbständigkeit und Lebensqualität muss im Vordergrund des Bemühens stehen, nicht unbedingt der buchhalterisch exakte Nachweis einzelner Ausgaben. Das Beispiel der Geschwisterfamilien zeigte zudem, wie wichtig die Einbindung des familiären und nachbarschaftlichen Umfeldes ist.

\footnotetext{
4 Der Rahmen für Arbeit und Beschäftigung von 4. Menschen vor allem mit geistiger Behinderung wird heute noch zu stark durch die Eingliederungshilfe als Sozialhilfeleistung sichergestellt. Die Möglichkeiten des Integrationsamtes nach dem SGB IX spielen deshalb nur eine geringe Rolle, weil dessen Förderungen nur im Zusammenhang mit dem allgemeinen Arbeitsmarkt möglich sind. Bietet der allgemeine Arbeitsmarkt wirklich keine Arbeitsplätze für Menschen mit geistiger Behinderung?
}

mit typisierten Leistungen befriedigt werden, die pauschal vergütet werden. Die Maßnahmepauschalen des SGB XII können zu landeseinheitlichen Fallpauschalen weiterentwickelt werden, wie wir sie nun in unseren Krankenhäusern haben. Dabei wird man sicher nicht zu den über 900 Fallkonstellationen des Krankenhausbereiches kommen müssen. Die Relativgewichte sollte man in der Behindertenhilfe leichter feststellen können als im Krankenhausbereich, wo man selbst für komplizierte Herztransplantationen Relativgewichte ermittelt hat.

Wir erleben heute in der Diskussion über die Finanzierung der stationären Behindertenhilfe eine Bandbreite von Argumenten, die von Vorschlägen für »am individuellen Bedarf orientierten Hilfesystemen « des Brüsseler Kreises bis zur Rückkehr zu Personalschlüsseln der 90er Jahre des vergangenen Jahrhunderts reichen, die einmal als obere Grenze vereinbart, von etlichen Einrichtungen aber nie umgesetzt worden waren und bei Berücksichtigung der heutigen Jahresarbeitszeit auch nicht mehr gerechtfertigt wären. Viele dieser Argumente sind geprägt vom Bemühen der Träger, die Arbeitsplätze ihrer Mitarbeiter zu sichern und die Arbeitsbedingungen zu erhalten oder zu verbessern.

Für mich steht fest, dass wir im Interesse der behinderten Menschen die »Institutionsbezogenheit« der Vergütung überwinden müssen. Die Vergütung, die Maßnahmepauschale, muss den behinderten Menschen mit seinem Hilfebedarf zum Maßstab von Pauschalen haben. Dann nämlich können wir uns auch in den Einrichtungen mehr um eine Qualität der Leistung kümmern, die vor allem Selbstbestimmung zum Ziel hat. 
Außerdem sollten wir darüber nachdenken, Rehabilitations- und Eingliederungserfolge zu belohnen, nicht aber — wie im derzeitigen System — zu bestrafen: Die niedrigere Hilfebedarfsgruppe führt zur niedrigeren Maßnahmepauschale! Das in der Jugendhilfe experimentierte Sozialraumbudget ging in die richtige Richtung, ist allerdings von Oberverwaltungsgerichten verworfen worden, weil es mit seinen Leistungen nicht gesetzeskonform war. Bei der Analyse der beiden Urteile ging mir unser »Wieslocher Klientenbudget « durch den Kopf, mit dem der Landeswohlfahrtsverband Baden zusammen mit Dr. Rath in Wiesloch experimentiert hat. Ich bin überzeugt, dass man unser damaliges Wieslocher Modell zu einem gesetzeskonformen Belohnungsmodell weiterentwickeln kann. Wir hätten in unserem heutigen Hilfebedarfsgruppensystem eine gute Datenbasis, um zu einer akzeptablen Durchschnittspauschale zu kommen, die bei einer Gesamtvereinbarung über das Leistungsspektrum vom betreuten Einzelwohnen bis zu Wohnheimbetreuung mit hohem Pflegeanteil als Maßnahmepauschale alles abdeckt. Man sollte dies in einer Stadt oder einem Landkreis zumindest bei der Hilfe für chronisch psychisch Kranke einmal experimentieren.

\section{Leistungen für Menschen mit Behinderung unabhän- gig von Sozialhilfe regeln}

Zur Selbstbestimmung kann auch eine weitgehende Unabhängigkeit von der Sozialhilfe führen, die als Fürsorgeleistung immer nachrangig sein muss, wenn sie dem Gedanken sozialer Gerechtigkeit noch entsprechen soll. Bei den vor uns liegenden, demografisch bedingten Fallzahlsteigerungen in der Eingliederungshilfe, die ohne Rechtsänderungen zwangsläufig zu erheblichen Ausgabesteigerungen führen werden, sollte auch der Gedanke an eine noch stärkere Berücksichtigung der Subsidiarität zum Beispiel bei der Sicherung des Lebensunterhaltes nicht tabu sein. Andererseits habe ich Verständnis für die Familien mit behinderten Kindern oder Geschwistern, die auf ihr besonderes Engagement hinweisen.

Daher halte ich den auch vom Deutschen Verein unterstützten Vorschlag zur Einführung eines »Bundesteilhabegeldes « für weiterführend. Mit dieser nicht nachrangigen Geldleistung in Form einer monatlichen Pauschale soll der besondere Aufwand von Menschen mit Behinderung für die Teilhabe am Leben in der Gemeinschaft ausgeglichen werden. Diese Pauschale kann dann als ein regelhaftes Persönliches Budget eingesetzt werden, um einzelne Teilhabemaßnahmen einzukaufen. Damit wäre teilweise eine Unabhängigkeit von der Sozialhilfe erreicht, die zusammen mit Leistungen der Pflegeversicherung, dem Werkstattlohn oder der Erwerbsunfähigkeitsrente nach langjähriger Werkstattbeschäftigung bis zur vollen Unabhängigkeit ausgebaut werden kann.

Mit dem Bundesteilhabegeld wäre auch der Bund wenn auch nur zu einem geringen Anteil — systemgerecht in die Finanzierung der gesamtgesellschaftlichen Aufgabe der Eingliederung behinderter Menschen in unsere Gesellschaft eingebunden. Die vorgeschlagene teilweise Refinanzierung ist sinnvoll und bewirkt, dass die finanziellen Konsequenzen für den Bundeshalthalt überschaubar sind. Ein allgemeines Bundesleistungsge- setz, wie es in der Vergangenheit gefordert wurde, wäre unter den Vorzeichen der heutigen Finanzpolitik sicher nicht realisierbar.

\section{Beteiligte bei großen Veränderungen mitnehmen}

Im Zusammenleben mit Menschen mit Behinderung steht die Gesellschaft vor großen Aufgaben. Die größte ist von den Nichtbehinderten zu bewältigen: eine Änderung in den Köpfen! Nicht Hilfe, sondern Begleitung selbstbestimmter, kompetenter Mitbürger! Diese Mitbürger leben in unserer Nachbarschaft, sie arbeiten mit und für uns, sie feiern mit uns. Wir ertragen ihre Besonderheiten, so wie sie unsere Eigenarten ertragen müssen. Wenn künftig die Politik in Bund, Ländern und Kommunen Maßnahmen zur Verbesserung der Situation von Menschen mit Behinderungen ergreifen will, sollte ein Kriterium im Vordergrund stehen: »Dienen diese Maßnahmen einem selbstbestimmten Leben?«

Bei dieser Veränderung müssen wir besorgte Eltern, aber auch die Mitarbeiter unserer Einrichtungen mitnehmen. Das kann nämlich für die berufliche Situation der Mitarbeiter große Änderungen bedeuten. Ich habe selbst ein solches »Change-Management « mit der Auflösung des Landeswohlfahrtsverbandes Baden hinter mir. Mehr als 400 Mitarbeiter wurden versetzt, teilweise an bis zu 200 Kilometer entfernt liegende Arbeitsplätze. Das war im Einzelfall nicht leicht. Aber unsere Mitarbeiter hatten - im Gegensatz zu vielen Mitarbeitern in Industrie und Handel — weiterhin einen sicheren Arbeitsplatz. Ähnlich sichere Arbeitsplätze hat auch die große Mehrheit der Mitarbeiter der Einrichtungen für Menschen mit Behinderung. Darauf dürfen wir in dieser Zeit der Veränderungen auch einmal hinweisen! 